\title{
Ultrasonographic, Surgical, and Histopathological Findings of a Uterine Leiomyoma in a Cow
}

\author{
Arvind Sharma, ${ }^{1}$ Adarsh Kumar, ${ }^{1}$ Sheikh Imran, ${ }^{1}$ Pankaj Sood, ${ }^{2}$ and Rajesh Kumar Asrani ${ }^{3}$ \\ ${ }^{1}$ Department of Veterinary Surgery and Radiology, College of Veterinary and Animal Sciences, CSK HP Agriculture University, \\ Himachal Pradesh, Palampur 176062, India \\ ${ }^{2}$ Department of Veterinary Gynaecology and Obstetrics, College of Veterinary and Animal Sciences, CSK HP Agriculture University, \\ Himachal Pradesh, Palampur 176062, India \\ ${ }^{3}$ Department of Veterinary Pathology, College of Veterinary and Animal Sciences, CSK HP Agriculture University, Himachal Pradesh, \\ Palampur 176062, India
}

Correspondence should be addressed to Sheikh Imran, sheikhimran_08@rediffmail.com

Received 28 November 2011; Accepted 15 January 2012

Academic Editor: L. Espino López

Copyright ( 2012 Arvind Sharma et al. This is an open access article distributed under the Creative Commons Attribution License, which permits unrestricted use, distribution, and reproduction in any medium, provided the original work is properly cited.

\begin{abstract}
The objective of this case report was to describe the ultrasonographic, surgical, and histopathological findings of a rare clinical case of uterine leiomyoma in a 5-year-old Holstein crossbred cow presented for diagnosis and treatment of infertility. Transrectal palpation revealed a large nonpainful mass suspected to be an abscess or a tumor in the caudal abdomen on the right side. Transabdominal ultrasonography revealed a round mass with irregular hypoechogenic/echogenic foci and a thin echogenic capsule around it. Ultrasonographic-guided centesis of the lesion under local analgesia did not yield any foul smelling aspirate leading to a tentative diagnosis of an intra-abdominal tumor. The lesion was later confirmed by exploratory laparotomy and histopathology as a case of uterine leiomyoma. The cow gave birth to a live normal calf 12 months following the surgery. Rectal examination after parturition revealed no evidence of the tumor at the surgical site. Ultrasonography enabled prompt, noninvasive diagnosis of uterine leiomyoma and proved to be a useful decision-making tool in the abdominal surgery of the cow. This is an interesting case which broadens the spectrum of the causes of infertility in cattle.
\end{abstract}

\section{Introduction}

A leiomyoma, histopathologically classified as mesenchymal tumor, is a benign neoplasia of smooth muscle. Leiomyomas of the genitalia occur far more frequently in females than males, and they are among the most commonly encountered tumors of the female reproductive system in almost all domestic species. In an abattoir study, leiomyomas were found to have a low frequency of occurrence as they represented only $1 \%$ to $2 \%$ of all neoplasia in sheep, cattle, and pigs [1]. Few case reports of vaginal and cervical leiomyomas in cows have been previously reported $[2,3]$; however, reports on ultrasonographic and surgical findings of uterine leiomyomas in cows have not been published in peer-reviewed journals until now. The purpose of this paper was to document a rare case of leiomyoma of the uterus in an infertile cow and her return to production following excision of the neoplasm.

\section{Case Presentation}

A 5-year-old Holstein crossbred cow was admitted to the Teaching Veterinary Clinic Complex, College of Veterinary and Animal Sciences in October 2009 with a history of repeat breeding for last 5 months. The cow had been vaccinated regularly and dewormed appropriately and had no other relevant medical history. The physical examination was normal, and results from a complete blood count (CBC) and biochemical profile were within normal limits. On transrectal palpation, the cervix, uterus, and both the ovaries were normal in size, consistency, and texture; however, detailed examination revealed a large nonpainful mass $(\sim 10 \mathrm{~cm}$ 


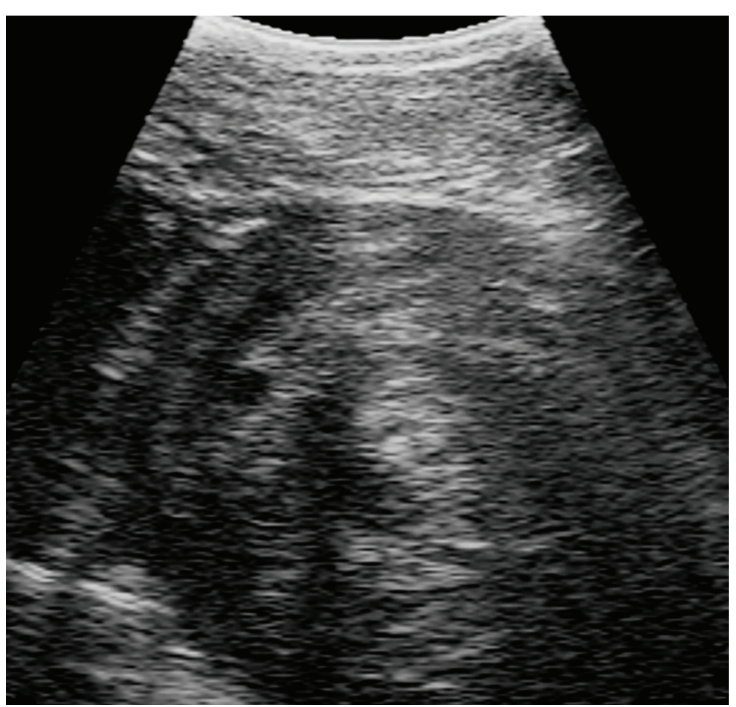

(a)

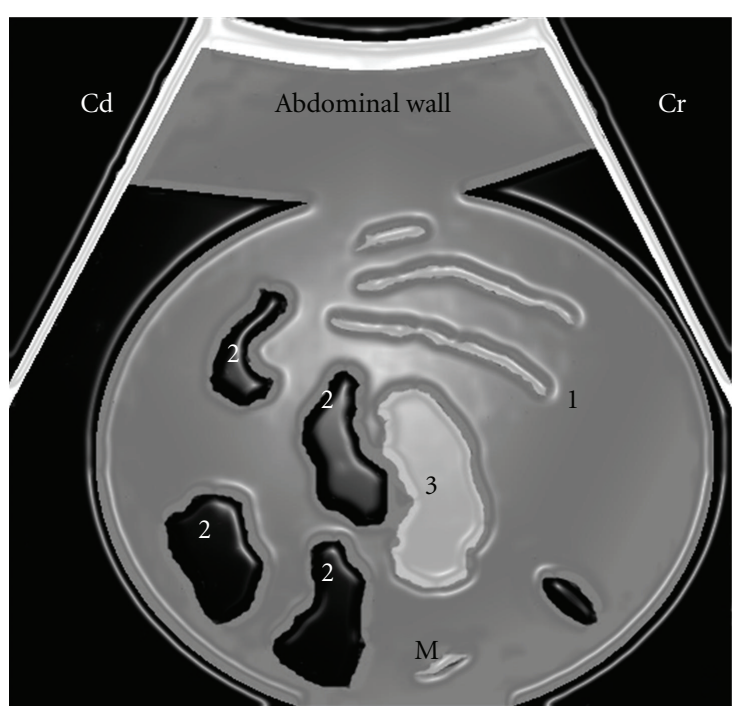

(b)

FIGURE 1: Ultrasonogram of the uterine tumour, imaged from the right paralumbar fossa, after bringing it closer to the abdominal wall by transrectal manipulation and placing the transducer parallel to the longitudinal axis of the cow. 1: uterine tumour, 2: hypoechogenic areas, 3: echogenic areas, Cd: caudal, Cr: cranial, and M: medial.

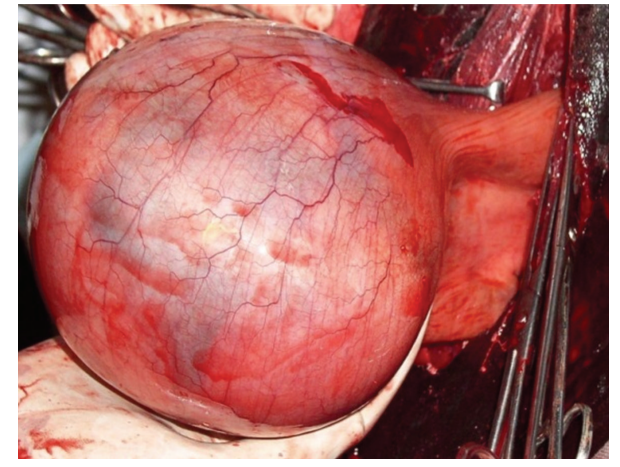

FIGURE 2: Uterine mass exteriorized after performing the right flank exploratory laparotomy.

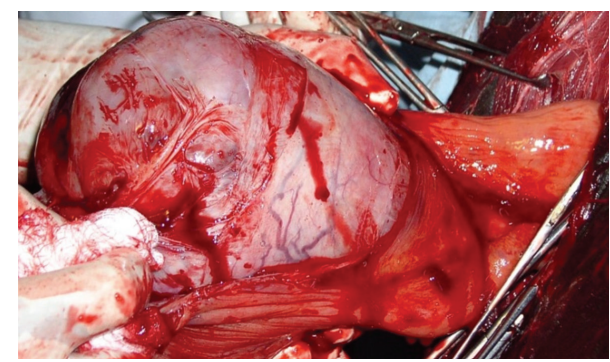

FIGURE 3: Hysterotomy depicting the intraluminal location of the mass in the right uterine horn.

diameter) suspected to be an abscess or a tumor in the caudal abdomen on the right side.

Transabdominal ultrasonography was done from right paralumbar fossa using a $3.5 \mathrm{MHz}$ curvilinear transducer with an assistant holding the mass transrectally towards abdominal wall. The lesion with a diameter of $7.8 \mathrm{~cm}$ appeared as a round mass with irregular hypoechogenic/echogenic foci and a thin echogenic capsule around it (Figure 1). Ultrasonographic-guided centesis of the lesion under local analgesia did not yield any foul smelling aspirate, which precluded the presence of an abscess, thereby hinting towards a possibility of an intra-abdominal tumor.

Standing caudal right flank celiotomy was recommended which would also allow the histological assessment. As expected, the pendulous mass was found attached to the uterine wall (Figure 2). A hysterotomy was made outside the abdomen to avoid contamination of the peritoneal cavity. Superficial incision revealed the intraluminal location of the mass (Figure 3). The uterine mass was excised from the right uterine horn and sent for the histological evaluation. Representative pieces of tissues from the tumor mass were collected in $10 \%$ neutral buffered formalin for histopathologic studies. Fixed tissues were trimmed, embedded in paraffin, sectioned at $3-5 \mu \mathrm{m}$, and stained with routine haematoxylin and eosin (H\&E) stain.

The uterine serosa and muscular layers were reapposed using a double inverting pattern with number 2 plain catgut. Sutures were placed only partial thickness incorporating the serosa and muscular layer of the uterus. The abdominal wound was lavaged and the incision closed routinely. The cow received general medical support including analgesics (Meloxicam (Melonex, Intas Pharmaceuticals Limited, India) at $0.5 \mathrm{mg} / \mathrm{kg}$ BW, IM, q24h for 3 days), antibiotics (Streptopenicillin (Dicrysticin-S LD, Zydus Animal Health Limited, India) at $2.5 \mathrm{gm}$, IM, q12h for 5 days), exercise with fresh water and electrolytes available at all times. The recovery of the cow was uneventful and was discharged 5 days postoperatively with no further medication but with instructions for no artificial insemination up to 2 months. 


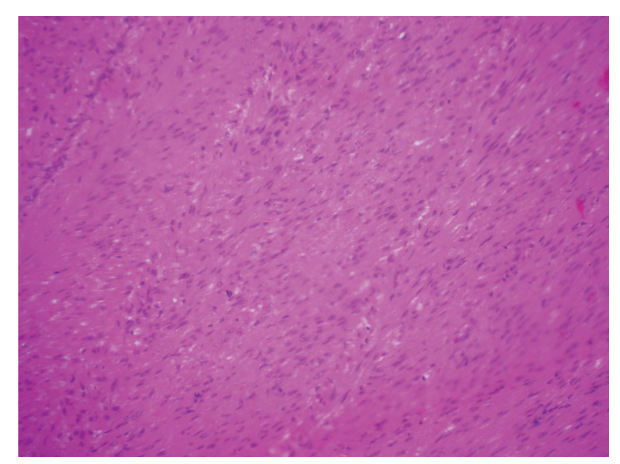

FIgUre 4: Photomicrograph depicting well-differentiated multiple layers of hypercellular and hyperchromatic interwoven bundles of smooth muscle cells. $\mathrm{H} \& \mathrm{E} \times 66$.

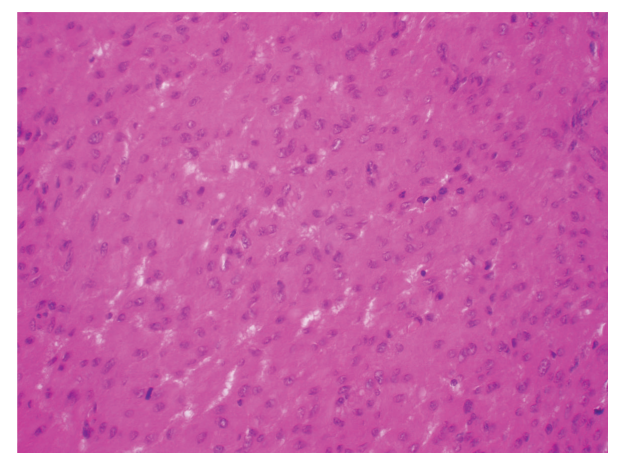

FIgURE 5: Photomicrograph from an area of tumor showing marked increase in its cellularity and occasional mitotic elements. $\mathrm{H} \& \mathrm{E} \times 132$.

Histopathological report of the excised uterine mass showed that it was a leiomyoma with hypercellular and hyperchromatic interlacing bundles of smooth muscle cells running in different layers (Figures 4 and 5). Mitotic figures were not uncommon. The tumor cells were uniform in size. In a few places, there were small areas of necrosis.

The past estrous activity of the cow was regular and periodic (interestrus interval of 19 to 22 days). However, the first estrus postsurgery was manifested after 40 days, with a regain in normal rhythm per se thereafter. During all overt estrous periods after surgery, the physical properties of genital discharge were normal.

\section{Discussion}

The etiology of the uterine leiomyoma is not known [3]. It consists of neoplastic cells of smooth muscle differentiation accompanied by varying quantities of connective tissue and lacks a glandular component $[4,5]$. Macroscopically, leiomyoma can reach up to $10-12 \mathrm{~cm}$ in diameter without being invasive. Initially, when the tumor is small, it has a fleshy consistency which becomes firm or even hard as it develops due to stromal connective tissue. In most cases, the leiomyoma projects like a nodular tumor into the uterine, vaginal, or cervical lumen [6].
The reason behind the cow's infertility in this case was uncommon in being associated with a progressive uterine growth. A logical explanation for this has been provided in case of a uterine leiomyoma in a mare [7]. A low-grade endometritis associated with uterine tumor, impairing of the implantation of the embryo due to mucosal damage caused by the tumor, and lastly an impediment to the transuterine migration of the embryo caused by the uterine mass itself could contribute to infertility.

Ultrasonographic-guided centesis was useful to reach at a tentative diagnosis; however, it could not ascertain the origin of the mass. Since the identification and histological typing of gastrointestinal tumours on the basis of the ultrasonographic appearance is not reliable, fine-needle aspiration or biopsy is almost always indicated when trying to establish a diagnosis [8]. The reason for not being able to pinpoint the origin of the mass ultrasonographically was the greater depth of the abdomen in the cow as the ultrasonographic waves gradually attenuated due to acoustic impedance of the tissues during the travel.

The future fertility of the cow was in question due to two possibilities-the recurrence of the tumor and the postoperative intra-abdominal adhesions around the uterus and ovaries. Adhesions affecting a section of or the entire reproductive tract are probably the most frequent sequelae of an ovariectomy, and they can have serious consequences on the future reproductive performance of the animal [9]. There are very few studies that have followed a large number of cows after surgery to determine postoperative fertility. In this case, the cow gave birth to a live normal calf 12 months following the surgery. Rectal examination after parturition revealed no evidence of the tumor at the surgical site.

In conclusion, ultrasonography enabled prompt, noninvasive diagnosis of the uterine leiomyoma and proved to be a useful decision-making tool in the abdominal surgery of the cow. This is an interesting case which broadens the spectrum of the causes of infertility in cattle.

\section{References}

[1] T. J. Hulland, "Tumors of muscle," in Tumors in Domestic Animals, J. E. Moulton, Ed., University of California Press, 2nd edition, 1978.

[2] R. O. Ramadan, W. S. Abu-El Fadle, A. M. El Hassan, M. Bindary, and A. Gomaa, "Vaginal leiomyoma in a cow," Reproduction in Domestic Animals, vol. 28, pp. 39-43, 1993.

[3] S. Sendag, Y. Cetina, M. Alana, F. Ilhana, F. Eskia, and A. Wehrendb, "Cervical leiomyoma in a dairy cattle," Animal Reproduction Science, vol. 103, pp. 355-350, 2008.

[4] R. S. Brodey and J. F. Roszel, "Neoplasms of the canine uterus, vagina, and vulva: a clinicopathologic survey of 90 cases," Journal of the American Veterinary Medical Association, vol. 151, no. 10, pp. 1294-1307, 1967.

[5] N. J. MacLachlan and P. C. Kennedy, "Tumors of the genital systems," in Tumors in Domestic Animals, D. J. Meuten, Ed., pp. 547-573, Iowa State Press, Ames, Iowa, USA, 4th edition, 2002.

[6] P. C. Kennedy and R. B. Miller, "The female genital system," in Pathology of Domestic Animals, K. V. F. Jubb, P. C. Kennedy, and N. Palmer, Eds., pp. 349-470, Academic Press, New York, NY, USA, 1993. 
[7] C. Berezowski, "Diagnosis of a uterine leiomyoma using hysteroscopy and a partial ovariohysterectomy in a mare," Canadian Veterinary Journal, vol. 43, no. 12, pp. 968-969, 2002.

[8] M. Frame, "Gastro-intestinal tract including pancreas," in Diagnostic Ultrasound in Small Animal Practice, P. Mannion, Ed., pp. 90-92, Blackwell, 2006.

[9] A. S. Turner and C. W. McIlwraith, Techniques in Large Animal Surgery, Lea and Febiger, Philadelphia, Pa, USA, 2nd edition, 1989. 

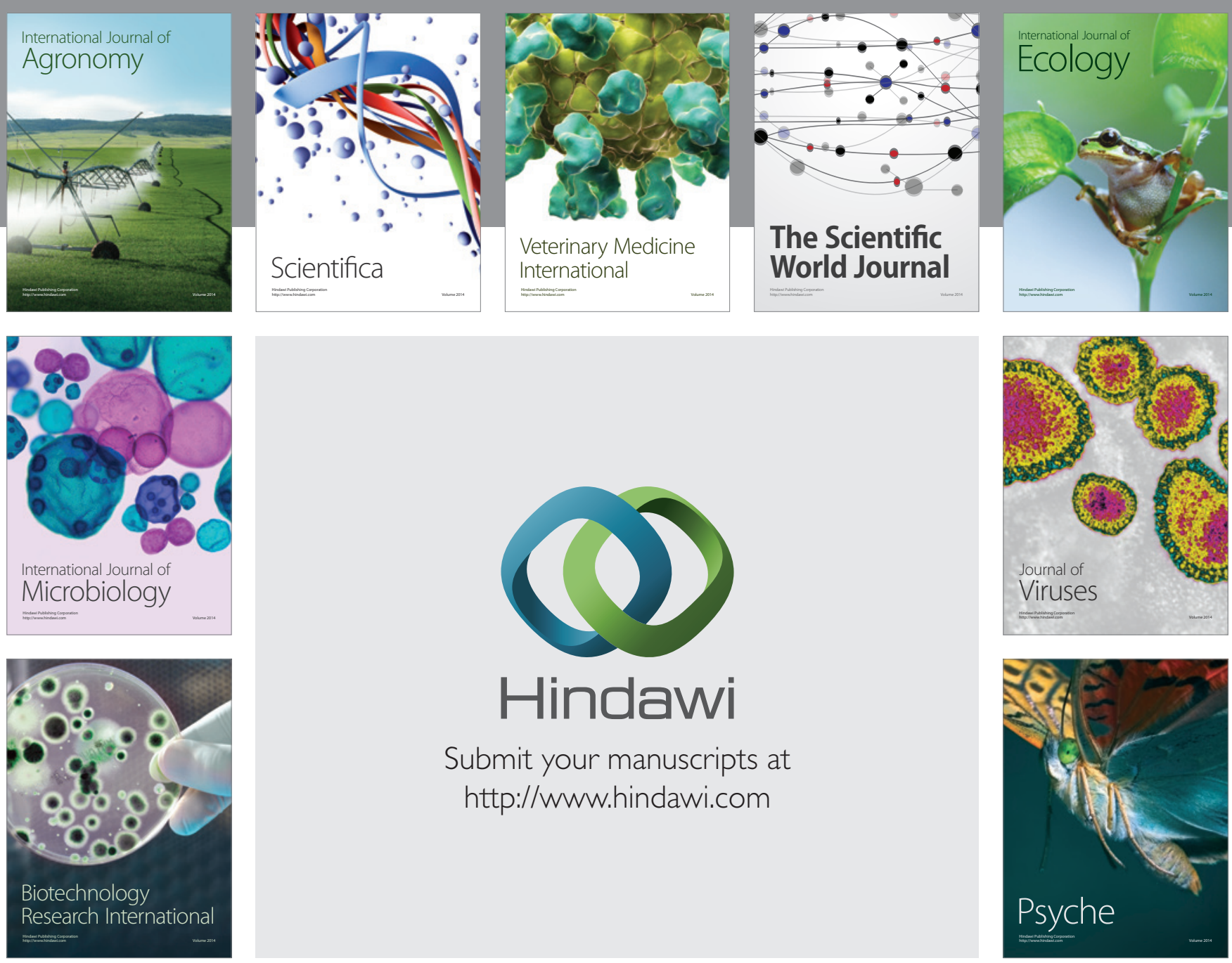

Submit your manuscripts at

http://www.hindawi.com
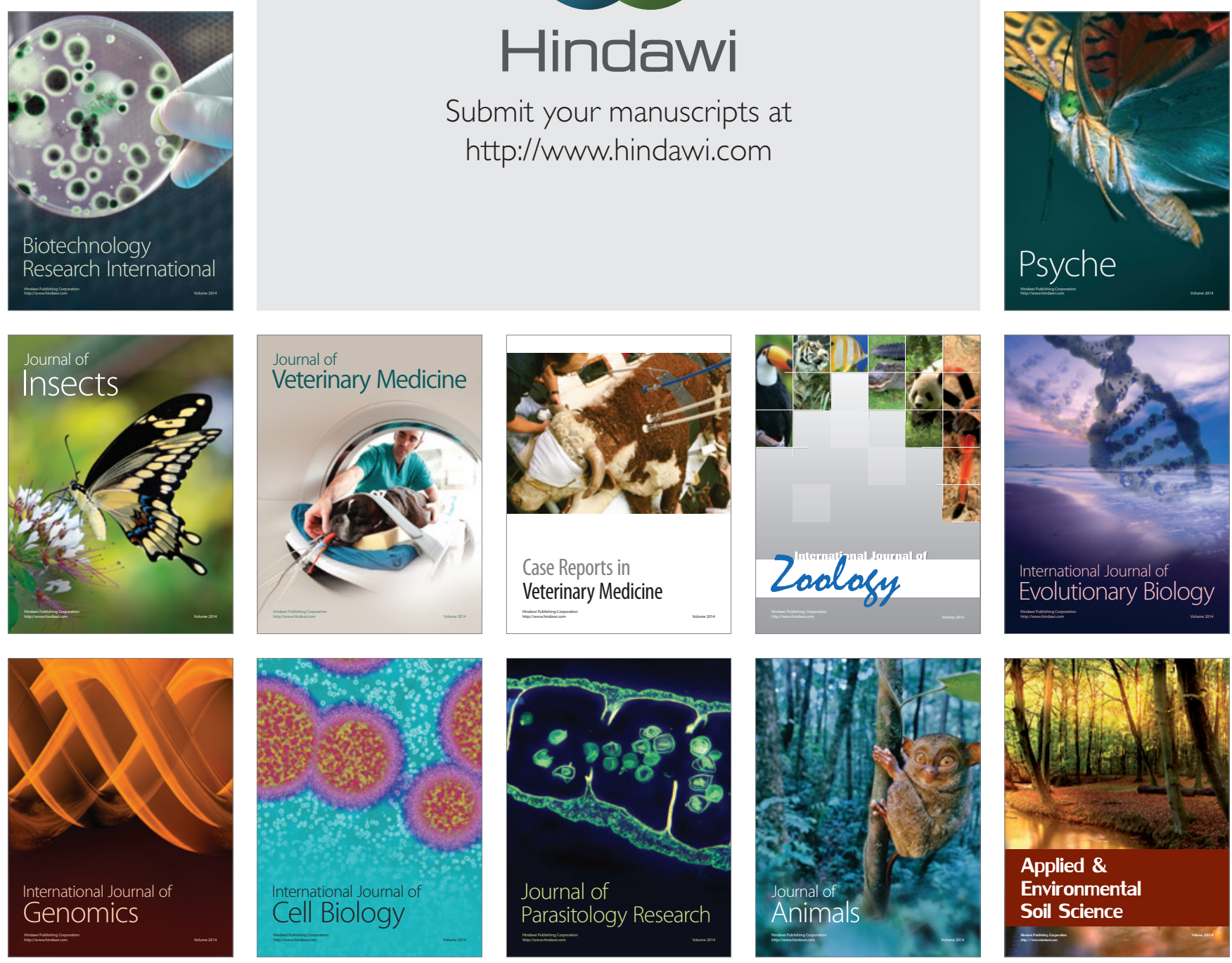